\title{
Grape seed proanthocyanidins inhibit proliferation of pancreatic cancer cells by modulating microRNA expression
}

\author{
WEIHUA WANG ${ }^{1,2^{*}}$, LEILEI ZHAN ${ }^{3 *}$, DONGQI GUO ${ }^{1,2}$, YANJU XIANG $^{1,2}$, MUXING TIAN $^{1,2}$, \\ YU ZHANG ${ }^{3}$, HONG WU $^{3}$, YAXUN WEI ${ }^{3}$, GANGLONG MA $^{3}$ and ZHANJIANG HAN ${ }^{1,4}$ \\ ${ }^{1}$ College of Life Science, Tarim University; ${ }^{2}$ Xinjiang Production and Construction Corps Key Laboratory of \\ Deep Processing of Agricultural Products in South Xinjiang, Alar, Xinjiang 843300; ${ }^{3}$ Center for Genome Analysis, \\ ABLife Inc., Wuhan, Hubei 430075; ${ }^{4}$ Xinjiang Production and Construction Corps Key Laboratory of Protection \\ and Utilization of Biological Resources in Tarim Basin, Alar, Xinjiang 843300, P.R. China
}

Received January 11, 2018; Accepted October 17, 2018

\section{DOI: $10.3892 /$ ol.2019.9887}

\begin{abstract}
MicroRNAs (miRNAs) are small non-coding RNAs of 18-25 nucleotides that modulate gene expression at the post-transcriptional level. Grape seed proanthocyanidins (GSPs), which are biologically active components in grape seeds, have been demonstrated to exhibit anticancer effects. The current study investigated whether GSPs can regulate miRNA expression and the possible anticancer molecular mechanisms of GSPs. Pancreatic cancer (PC) cell samples, SS3, SS12 and SS24, were treated with $20 \mu \mathrm{g} / \mathrm{ml}$ GSPs for 3, 12 and 24 h, respectively. Control samples, SC3, SC12 and SC24, were also prepared. Using miRNA-seq, transcriptome analysis identified 24, 83 and 83 differentially expressed (DE) miRNAs in SS3 vs. SC3, SS12 vs. SC12 and SS24 vs. SC24, respectively. This indicated that treatment with GSPs could modulate the expression of miRNAs. Subsequently, 74, 598 and 1,204 target genes for the three sets of DE miRNAs were predicted. Gene Ontology and Kyoto Encyclopedia of Genes and Genomes analysis revealed that multiple target genes were associated with the proliferation and apoptosis of PC cells. In addition, a network was constructed of the DE miRNAs and the target genes associated with PC. The associations identified suggested that treatment with GSPs may inhibit the proliferation of PC cells through the modulation of miRNA expression.
\end{abstract}

Correspondence to: Professor Zhanjiang Han, College of Life Science, Tarim University, 705 Hongqiao South Road, Alar, Xinjiang 843300, P.R. China

E-mail: hanzhanjiang@163.com

*Contributed equally

Key words: microRNAs, grape seed proanthocyanidins, pancreatic cancer cells, microRNA sequencing, proliferation

\section{Introduction}

MicroRNAs (miRNAs) are small single-stranded non-coding RNAs, consisting of no more than 25 nucleotides (nts) cleaved from hairpin pre-miRNA precursors (1). miRNAs serve important roles in the epigenetic regulation of protein expression, are encoded by their respective genes and are highly conserved in all multicellular eukaryotes and certain unicellular eukaryotes (2). Generally, miRNAs recognize and bind to complementary 3'-untranslated regions of target mRNAs, which can induce degradation or transcriptional repression of the target (3). However, it has also been revealed that miRNAs can upregulate the translation of target genes by interacting with regulatory complexes (4). A single miRNA has the capacity to regulate the expression of multiple target mRNAs and one gene can be modulated by multiple miRNAs (5). A number of miRNAs have been identified in multiple eukaryotic organisms; a total of 2,588 human genome miRNAs are included in the miRBase database (6). It is estimated that $>60 \%$ of all mRNAs are modulated by miRNAs at the post-transcriptional level (7). Previous studies have identified that miRNAs serve critical roles in numerous biological processes, including development, cell differentiation, proliferation and apoptosis (8). Furthermore, miRNA-associated dysfunction is associated with a number of diseases, including Alzheimer's disease (9), cardiovascular diseases (10) and numerous cancer types (11). Additionally, the modulation of miRNAs has been demonstrated to provide therapeutic benefits. For example, miRNAs have been demonstrated to be useful in cancer therapy (12).

Grape seed proanthocyanidins (GSPs), biologically active components that make up $70-95 \%$ of the proanthocyanidins, have been reported to modulate the expression of certain miRNAs that serve important roles in cancer, glucose homeostasis and lipid homeostasis $(13,14)$. Previous studies have revealed that GSPs exhibit inhibitory effects in various cancer types, including pancreatic cancer (PC) (15-17). However, to the best of our knowledge, the molecular mechanism of this anticancer effect remains unknown. In our previous study transcriptome analysis of the response of PC cells to treatment with GSPs was performed using RNA-seq (18). This indicated 
that the expression levels of multiple genes associated with the proliferation of PC cells were altered in GSPs-treated cells. However, to the best of our knowledge, it remains to be investigated whether treatment with GSPs can regulate the expression of miRNAs in PC cells.

To determine how treatment with GSPs regulates the expression of miRNA in PC cells the current study generated PC cell samples (SS3, SS12 and SS24) treated with $20 \mu \mathrm{g} / \mathrm{ml} \mathrm{GSPs}$ for 3, 12 and $24 \mathrm{~h}$, respectively. Subsequently, miRNA-seq experiments were performed. Compared with the miRNA data obtained from control cell samples (SC3, SC12 and SC24), numerous differentially expressed (DE) miRNAs were identified in SS3, SS12 and SS24. In addition, a number of target genes of the DE miRNAs were identified. Analysis using the Gene Ontology (GO) and Kyoto Encyclopedia of Genes and Genomes (KEGG) databases revealed that multiple target genes were enriched in functional pathways associated with the proliferation of cancer cells. This suggested that treatment with GSPs may exhibit inhibitory effects on cancer cells through the regulation of miRNAs.

\section{Materials and methods}

Cell culture and reagents. The human PC cell line PANC-1 was obtained from Procell (Wuhan, China) (http://www.procell. com.cn/) and cultured in monolayers in Dulbecco's modified Eagle's medium (Invitrogen; Thermo Fisher Scientific, Inc., Waltham, MA, USA) supplemented with $10 \%$ fetal bovine serum (Hyclone; GE Healthcare Life Sciences, Logan, UT, USA) in a humidified incubator at $37^{\circ} \mathrm{C}$ and $5 \% \mathrm{CO}_{2}$. The GSP extract, obtained from JF-NATURAL (Tianjin, China; catalog no. J011003), contained monomeric (9.5\%), dimeric $(12.8 \%)$, trimeric $(76.7 \%)$ and oligomeric $(1 \%)$ procyanidins. The $100 \mu \mathrm{g}$ GSPs were dissolved in $100 \mu 1$ dimethylsulfoxide (DMSO; Sigma-Aldrich; Merck KGaA, Darmstadt, Germany) for $10 \mathrm{~min}$ at room temperature prior to addition to cell culture media. The maximum concentration of DMSO in the media did not exceed $0.1 \%$. PANC-1 cells were treated with $20 \mu \mathrm{g} / \mathrm{ml}$ GSP for 3,12 and $24 \mathrm{~h}$ at $37^{\circ} \mathrm{C}$, and the treated cells were used to prepare for SS3, SS12 and S24 samples, respectively. Additionally, control cell samples were treated with DMSO for 3,12 and $24 \mathrm{~h}$ at $37^{\circ} \mathrm{C}$, and then $\mathrm{SC} 3, \mathrm{SC} 12$ and $\mathrm{SC} 24$ samples were prepared accordingly.

Cell viability assay. GSP-treated PANC-1 cells were plated in 96-well cell culture plates at $5 \times 10^{3}$ cells/well and incubated for $24 \mathrm{~h}$ at $37^{\circ} \mathrm{C}$. Subsequently, $50 \mu \mathrm{l}$ of MTT solution $(5 \mathrm{mg} / \mathrm{ml}$; Sigma-Aldrich; Merck KGaA) was added to each well and the cells were incubated for a further $4 \mathrm{~h}$ at $37^{\circ} \mathrm{C}$. Following $3 \mathrm{~min}$ centrifugation $(5500 \mathrm{xg})$ at $4^{\circ} \mathrm{C}$, the supernatant was removed from each well. The formazan crystals produced from MTT in each well were dissolved in $150 \mu \mathrm{l}$ DMSO and the optical density values were measured at $490 \mathrm{~nm}$.

Flow cytometry. GSP-induced apoptosis in PC cells was determined by flow cytometry using the Annexin V-fluorescein isothiocyanate (FITC) Apoptosis Detection kit (BD Biosciences, Franklin Lakes, NJ, USA). Following treatment with GSPs for $48 \mathrm{~h}, 2 \times 10^{5}$ cells were harvested, washed twice with PBS and incubated with Annexin V-FITC and propidium iodide for $10 \mathrm{~min}$ in the dark at room temperature. The stained cells were then detected and analyzed by the MoFlo XDP flow cytometer (Beckman Coulter, Inc., Brea, CA, USA) and Cell Quest 3.3 software (BD Biosciences, Franklin Lakes, NJ, USA).

RNA extraction and small RNA sequencing. Total RNA was extracted from PC cells using TRIzol reagent (Invitrogen; Thermo Fisher Scientific, Inc.), according to the manufacturer's protocol. Subsequently, RQ1 DNase (Promega Corporation, Madison, WI, USA) was used to remove DNA. The quality and quantity of the purified RNA was monitored by absorbance at 260 and $280 \mathrm{~nm}$, and the A260: A280 ratio, using a SmartSpec Plus Spectrophotometer (Bio-Rad Laboratories, Inc., Hercules, CA, USA). RNA integrity was further verified by electrophoresis of a $1.5 \%$ agarose gel.

Total RNA (3 $\mu \mathrm{g}$ ) from each sample was used for small RNA cDNA library preparation with a Balancer NGS Library Preparation kit (Gnomegen, San Diego, CA, USA), according to the manufacturer's protocol. The whole library was subjected to $10 \%$ native polyacrylamide gel electrophoresis and the bands corresponding to miRNA insertion were cut and eluted. Following ethanol precipitation and washing, the purified libraries were quantified using the QubitFluorometer (Invitrogen; Thermo Fisher Scientific, Inc.). These small RNA libraries were applied to NextSeq 500 (Illumina, Inc., San Diego, CA, USA) $76 \mathrm{nt}$ pair-end sequencing.

Identification of conserved and novel miRNA. To obtain reliable clean reads, raw reads were processed by the FASTX-Toolkit (version 0.0.13)(http://hannonlab.cshl.edu/fastx_toolkit/). During this procedure, adaptor sequences and low quality tags were discarded. Based on the length of the mature miRNA and adapter lengths, RNAs $<16$ or $>30$ nts in length were also excluded from further analysis. The high-quality clean reads were subsequently searched against the Rfam database (version 12.0) (http://rfam.xfam.org/) using Bowtie (19). Matches to ribosomal RNAs and transfer RNAs were excluded. Subsequently, the remaining unique sequences were aligned against the miRBase database (20) using Bowtie, with one mismatch allowed. The matched small RNA sequences were considered to be conserved miRNAs and the unaligned sequences were potential candidates for novel miRNAs. To identify novel miRNAs, the unique sequences were aligned to the human reference genome sequence (GRCH38) using the miRDeep algorithm (21).

Bioinformatics analysis. To investigate the expression profiles of identified miRNAs, the frequency of miRNA counts were normalized to transcripts per million (TPM) using the following formula: Normalized expression=actual read count/total read count $\times 10^{6}$. DE miRNAs were analyzed using the edgeR (v3.22) package of Bioconductor software (22). A fold change (FC) $\leq 2$ or $<0.5$ and $\mathrm{P} \leq 0.01$ indicated a statistically significant DE miRNA. The miRanda algorithm (23) was used on human miRNA and transcript sequences of miRBase and TargetScan (7) for the prediction of putative miRNA targets.

To predict the gene function and calculate the frequency distribution of functional categories, GO and KEGG analysis were employed using the DAVID bioinformatics database (24). 
Networks were constructed by calculating the Pearson's correlation coefficient (PCC) for the expression levels of DE miRNAs and target genes. Cytoscape (version 3.0.2) was used to display the co-expression network (25).

Validation of the target genes by reverse transcription-quantitative polymerase chain reaction $(R T-q P C R)$. To validate the RNA-seq data, RT-qPCR was performed for selected target genes and normalization was achieved with the human reference gene GAPDH. The primers used are presented in Table I. The same RNA samples for RNA-seq were used for RT-qPCR, and RNA extraction was performed with the same protocol and materials. In each pooled sample, $1 \mu \mathrm{g}$ of total RNA was reverse transcribed using the PrimeScript ${ }^{\mathrm{TM}} \mathrm{RT}$ Reagent kit (Takara Bio, Inc., Otsu, Japan), according to the manufacturer's protocol. qPCR was performed with the S1000 Thermal Cycler (Bio-Rad Laboratories, Inc.) and Bestar SYBR Green RT-PCR Master mix (DBI Bioscience, Shanghai, China). The following thermocycling conditions were used: $95^{\circ} \mathrm{C}$ for $10 \mathrm{~min}, 38$ cycles of $95^{\circ} \mathrm{C}$ for $15 \mathrm{sec}$ and $60^{\circ} \mathrm{C}$ for $1 \mathrm{~min}$. PCR amplifications were performed in triplicate for each sample, and the results were quantified by $2^{-\Delta \Delta \mathrm{Cq}}$ methods (26).

Statistical analysis. All data are presented as the mean \pm standard deviation. For comparisons between two groups, statistically significant differences between means were identified by Student's t-test. For multiple comparisons, the significance was determined by one-way analysis of variance followed by a Tukey's post hoc test. $\mathrm{P}<0.05$ was considered to indicate a statistically significant difference.

Online data deposition. The datasets obtained in the current study were deposited in the National Center for Biotechnology Information Gene Expression Omnibus database under the accession number GSE107409. The transcriptome database (GSE85610) generated in our previous study (18) was also deposited.

\section{Results}

$G S P$ s repressed the viability of $P C$ cells in vitro. Using flow cytometry, the current study identified that treatment with 0 , 20,40 or $60 \mu \mathrm{g} / \mathrm{ml}$ GSP significantly induced apoptosis of the PC cell line PANC-1 in a dose-dependent manner (Fig. 1A and $\mathrm{B}$ ). In addition, the effect of treatment with GSPs on the viability of PC cells was investigated by MTT assay. A significant dose-dependent decrease in the viability of PC cells was identified $24 \mathrm{~h}$ after treatment (Fig. 1C). The aforementioned apoptosis and cell viability results were in accordance with previous studies (15-17). Furthermore, it was identified that treatment with GSPs was associated with necrosis in a small number of of PC cells in a dose-dependent manner (Fig. 1A).

miRNA expression profiles in PC cells treated with GSPs. To avoid high levels of necrotic cells and investigate how GSPs modulate the expression of miRNAs in PC cells, PANC-1 cells were treated with $20 \mu \mathrm{g} / \mathrm{ml} \mathrm{GSP}$ for 3,12 and $24 \mathrm{~h}$, and the miRNA samples termed SS3, SS12 and SS24 were prepared, respectively. Cells treated with DMSO for 3, 12 and $24 \mathrm{~h}$ served as controls, and the control miRNA samples termed SC3,
SC12 and SC24 were prepared, respectively. Two biological replicates were generated at each time point, therefore, a total of 12 small RNA libraries (SS3-A, SS3-B, SC3-A, SC3-B; SS12-A, SS12-B, SC12-A, SC12-B; SS24-A, SS24-B, SC24-A and SC24-B) were constructed for miRNA-seq.

Using Illumina NextSeq 500, >89.38 million reads were generated, corresponding to $\sim 7.44$ million sequence reads per sample and $\sim 70 \%$ of all reads were successfully mapped against the current human reference genome (Table II). In addition, the proportion of clean reads mapped to the Rfam database for each sample is presented in Table III. In total, $>79.6 \%$ of reads were matched to Rfam and multiple mapped reads were $>84.2 \%$.

The clean reads were mapped to miRBase and it was identified that $39.3 \%$ reads were matched to mature miRNAs (Table IV). The read counts were normalized to TPM and a total of 2,578 miRNAs were revealed, consisting of 502 novel miRNAs (data not shown).

Determination of DE miRNAs. DE miRNAs were determined using edgeR (22) and the following criteria: $\mathrm{P} \leq 0.01$ and $\mathrm{FC} \geq 2$ or $\leq 0.5$. A total of 24,83 and 83 DE miRNAs were identified in SS3 vs. SC3, SS12 vs. SC12 and SS24 vs. SC24, respectively (Fig. 1D and E). The highest and lowest $\mathrm{FC}$ values were $2 \times 10^{8.59}$ and $2 \times 10^{-8.12}$, respectively (data not shown). These findings indicated that treatment with GSPs is associated with the expression levels of numerous miRNAs in PANC-1 cells. In addition, only three common DE miRNAs were identified between all three comparisons, suggesting that treatment with GSPs can regulate the expression levels of different miRNAs depending on the duration of treatment.

Prediction of DE miRNA target genes. miRNAs commonly exert their functions by binding to complementary target sites in the mRNAs of their target genes. Using the miRanda algorithm (23) on human miRNA and transcript sequences of miRBase and TargetScan (7), 74, 598 and 1,204 target genes were identified for the DE miRNAs in SS3 vs. SC3, SS12 vs. SC12 and SS24 vs. SC24, respectively (Fig. 2). This suggests that the DE miRNAs have the capacity to regulate the expression levels of multiple genes.

In our previous study, PANC-1 cells were also treated with $20 \mu \mathrm{g} / \mathrm{ml}$ GSPs for 3, 12 and $24 \mathrm{~h}$, and $12 \mathrm{cDNA}$ libraries (S3-A, S3-B, C3-A, C3-B; S12-A, S12-B, C12-A, C12-B; S24-A, S24-B, C24-A and C24-B) were constructed for RNA-seq. The current study analyzed the expression levels of the predicted target genes using the transcriptome database generated in our previous study (GSE85610). This revealed that all predicted target genes of the DE miRNAs were downregulated or upregulated following treatment with GSPs, suggesting that GSPs may modulate gene expression by the regulation of miRNAs. The expression levels determined by RT-qPCR of certain target genes, including MutS homolog 6, epidermal growth factor receptor, cyclin dependent kinase 6 and DNA methyltransferase 1, were in accordance with the RNA-seq results (Fig. 3).

Functional analysis of target genes. To identify the pathways associated with the target genes of the DE miRNAs, GO enrichment analysis was first conducted (Fig. 4). It was demonstrated that 7, 77 and 124 significant functional terms were identified 
Table I. Genes and primers used for reverse transcription-quantitative polymerase chain reaction.

\begin{tabular}{lll}
\hline Gene & \multicolumn{1}{c}{ Forward primer (5'-3') } & \multicolumn{1}{c}{ Reverse primer (5'-3') } \\
\hline CDK6 & GCAGCTTCTCTTCTTCTGGAAT & TGTGGAGGATTGCTATCTGGAG \\
MSH6 & GTCCTATGTGTCGCCCAGTA & TTCCTGCTCCTCTTCCTCAC \\
EGFR & GTGTGCCCTGTAACCTGAC & GTGACTGAACATAACTGTAGGC \\
DNMT1 & CCGTGGATGAGGACCTGTAC & CCTGCCGTTGCTCTTCTTG \\
GAPDH & GGTCGGAGTCAACGGATTTG & GGAAGATGGTGATGGGATTC
\end{tabular}

CDK6, cyclin dependent kinase 6; MSH6, MutS homolog 6; EGFR, epidermal growth factor receptor; DNMT1, DNA methyltransferase 1.

A
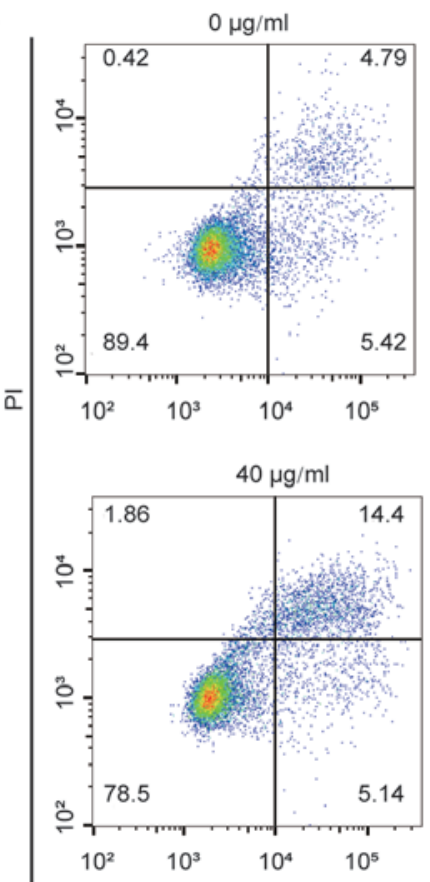
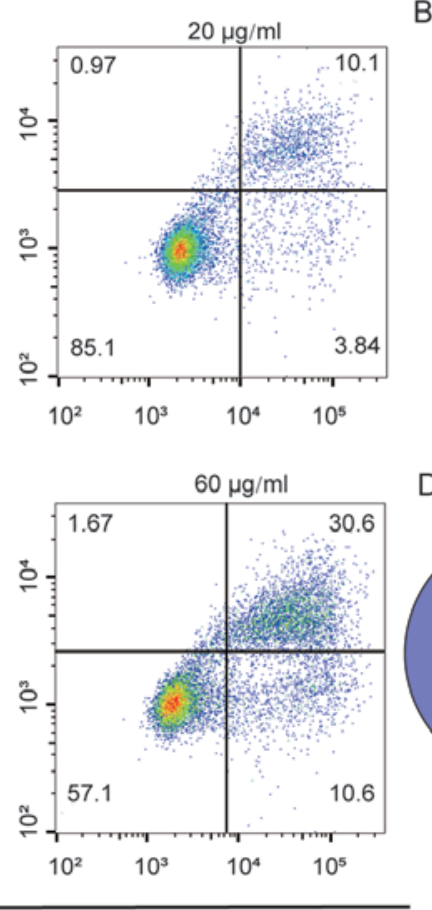
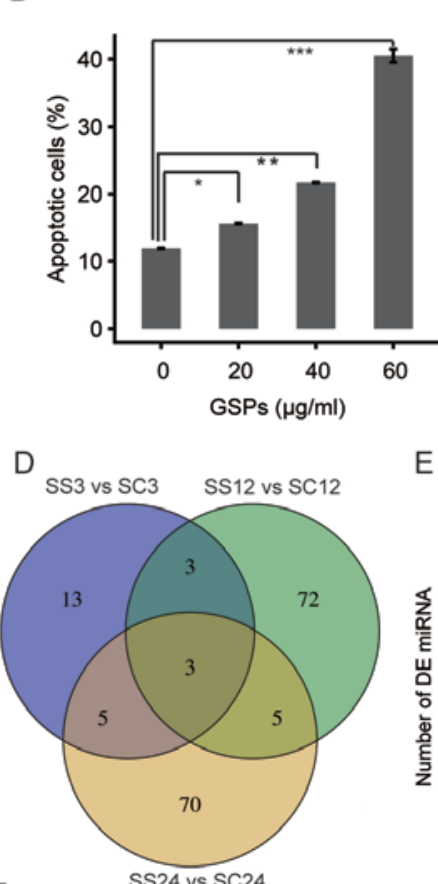

$\mathrm{E}$
C
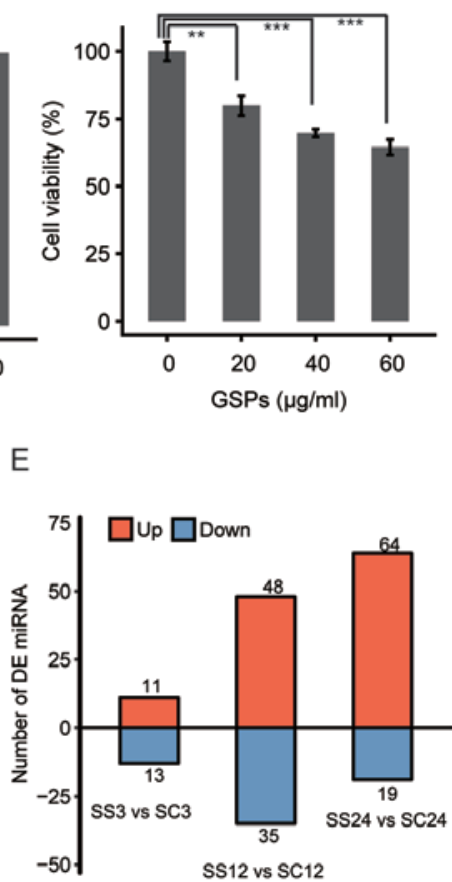

Annexin V/FITC

Figure 1. Determination of DE miRNAs in PC cells following treatment with GSPs. (A) GSPs induce apoptosis of the PC cell line PANC-1. Cells were treated with different concentrations of GSPs and harvested at $24 \mathrm{~h}$ post-treatment for assessment of apoptosis using Annexin V-fluorescein isothiocyanate staining coupled with flow cytometry. The upper left, upper right and lower left quadrants represent necrotic, late apoptotic and early apoptotic events, respectively. (B) Total percentage of apoptotic PC cells in each treatment group are quantified with data presented as the mean \pm SD of two independent experiments. (C) Treatment with GSPs reduces the viability of PC cells. Viability of the PC cells was determined by MTT assay. The data are reported as the percentage relative to control cells and presented as the mean \pm SD of six replicates. (D) Venn diagrams of the DE miRNAs identified in the SS3 vs. SC3, SS12 vs. SC12 and SS24 vs. SC24 comparisons. (E) The number of upregulated and downregulated DE miRNAs in the three comparisons. Data are presented as the mean \pm SD. Statistical analysis was performed using one-way analysis of variance followed by Tukey's post hoc test. ${ }^{*} \mathrm{P}<0.05,{ }^{* * *} \mathrm{P}<0.01,{ }^{* * *} \mathrm{P}<0.001$. DE, differentially expressed; miRNA, microRNA; GSP, grape seed proanthocyanidin; PC, pancreatic cancer; SD, standard deviation; PI, propidium iodide; PE, phycoerythrin.

for the target genes of the DE miRNAs in SS3 vs. SC3, SS12 vs. SC12 and SS24 vs. SC24, respectively (P<0.05; Fig. 4A, $\mathrm{C}$ and E). Multiple target genes were enriched in 'positive regulation of cell proliferation' (GO: 0008284) and 'negative regulation of apoptotic process' (GO: 0043066), which indicates that the DE miRNAs induced by treatment with GSPs may serve a regulatory role in the proliferation and apoptosis of PANC-1 cells. Targeting factors associated with cell cycle is a potential approach for cancer therapy; therefore, GSPs may exhibit inhibitory effects on the proliferation of cancer cells.

In addition, KEGG enrichment analysis was performed. It was revealed that 15,42 and 47 significant functional terms were identified for the target genes of the DE miRNAs in
SS3 vs. SC3, SS12 vs. SC12 and SS24 vs. SC24, respectively $(\mathrm{P}<0.05)$. Although 'microRNAs in cancer' (ID: hsa05206) and 'pancreatic cancer' (ID: hsa05212) were not presented in Fig 4 (only top10 terms were presented), numerous target genes were enriched in them and 'pathways in cancer' (ID: hsa05200). This suggests that treatment with GSPs may exhibit anticancer effects by disrupting the functional pathways of PC cells.

Integrative analysis of DE miRNA and mRNA expression. The aforementioned results revealed that the target genes of certain DE miRNAs were associated with the regulation of cancer. To further investigate this, a co-expression network was constructed according to PCC values that indicated the 
Table II. Small RNA-Seq results.

\begin{tabular}{|c|c|c|c|c|}
\hline Sample & Raw data & Clean reads & Total mapped reads & Unique mapped reads \\
\hline SC3-A & 8764786 & 6564553 & 6344960 & 4373569 \\
\hline SC3-B & 5900986 & 4283482 & 4141642 & 2896531 \\
\hline SC12-A & 7603914 & 4836317 & 4456413 & 2834055 \\
\hline SC12-B & 4918096 & 3034397 & 2614249 & 1657090 \\
\hline SC24-A & 8437896 & 6022757 & 5777249 & 4000517 \\
\hline SC24-B & 7878026 & 5703819 & 5467646 & 3774752 \\
\hline SS3-A & 7470866 & 5732424 & 5538865 & 3835541 \\
\hline SS3-B & 8821086 & 7070260 & 6855861 & 4833465 \\
\hline SS12-A & 6258112 & 5002508 & 4760228 & 3293693 \\
\hline SS12-B & 8683604 & 6627072 & 6366737 & 4431579 \\
\hline SS24-A & 8985184 & 7399980 & 7002763 & 4752743 \\
\hline SS24-B & 5657588 & 3457776 & 3328007 & 2009410 \\
\hline
\end{tabular}

Table III. Clean reads mapped to Rfam.

\begin{tabular}{lcccc}
\hline Sample & Input reads & Total mapped reads & Unique mapped reads & Multiple mapped reads \\
\hline SC3-A & 6564553 & 5412203 & 808375 & 4603828 \\
SC3-B & 4283482 & 3538961 & 529044 & 3009917 \\
SC12-A & 4836317 & 3663218 & 567185 & 3096033 \\
SC12-B & 3034397 & 2082789 & 350653 & 1732136 \\
SC24-A & 6022757 & 4818716 & 780206 & 4038510 \\
SC24-B & 5703819 & 4574160 & 726961 & 3847199 \\
SS3-A & 5732424 & 4752397 & 718256 & 4034141 \\
SS3-B & 7070260 & 5883811 & 906572 & 4977239 \\
SS12-A & 5002508 & 4013582 & 644253 & 3369329 \\
SS12-B & 6627072 & 5256520 & 846982 & 4409538 \\
SS24-A & 7399980 & 5708723 & 930190 & 4778533 \\
SS24-B & 3457776 & 2669152 & 423987 & 2245165 \\
\hline
\end{tabular}

Table IV. Clean reads mapped against mature microRNAs of miRBase.

\begin{tabular}{|c|c|c|c|c|}
\hline Sample & Input reads & Total mapped reads & Unique mapped reads & Multiple mapped reads \\
\hline SC3-A & 6564553 & 2762812 & 2629156 & 133656 \\
\hline SC3-B & 4283482 & 1781052 & 1694783 & 86269 \\
\hline SC12-A & 4836317 & 1710715 & 1618013 & 92702 \\
\hline SC12-B & 3034397 & 1032329 & 980273 & 52056 \\
\hline SC24-A & 6022757 & 2302503 & 2190377 & 112126 \\
\hline SC24-B & 5703819 & 2230508 & 2123120 & 107388 \\
\hline SS3-A & 5732424 & 2420577 & 2299253 & 121324 \\
\hline SS3-B & 7070260 & 3110970 & 2944227 & 166743 \\
\hline SS12-A & 5002508 & 1922143 & 1828317 & 93826 \\
\hline SS12-B & 6627072 & 2534886 & 2396862 & 138024 \\
\hline SS24-A & 7399980 & 2816014 & 2673813 & 142201 \\
\hline SS24-B & 3457776 & 1236899 & 1166154 & 70745 \\
\hline
\end{tabular}

strength of the correlation between the expression levels of the DE miRNAs and the target genes that were enriched in 'pathways in cancer' (ID: hsa05200), 'microRNAs in cancer'
(ID: hsa05206) and 'pancreatic cancer' (ID: hsa05212) (PPC $\geq 0.6, \mathrm{P}<0.01$; Fig. 5). In total, $36 \mathrm{DE}$ miRNAs and 66 target genes were analyzed, and 26 DE miRNAs were 
A

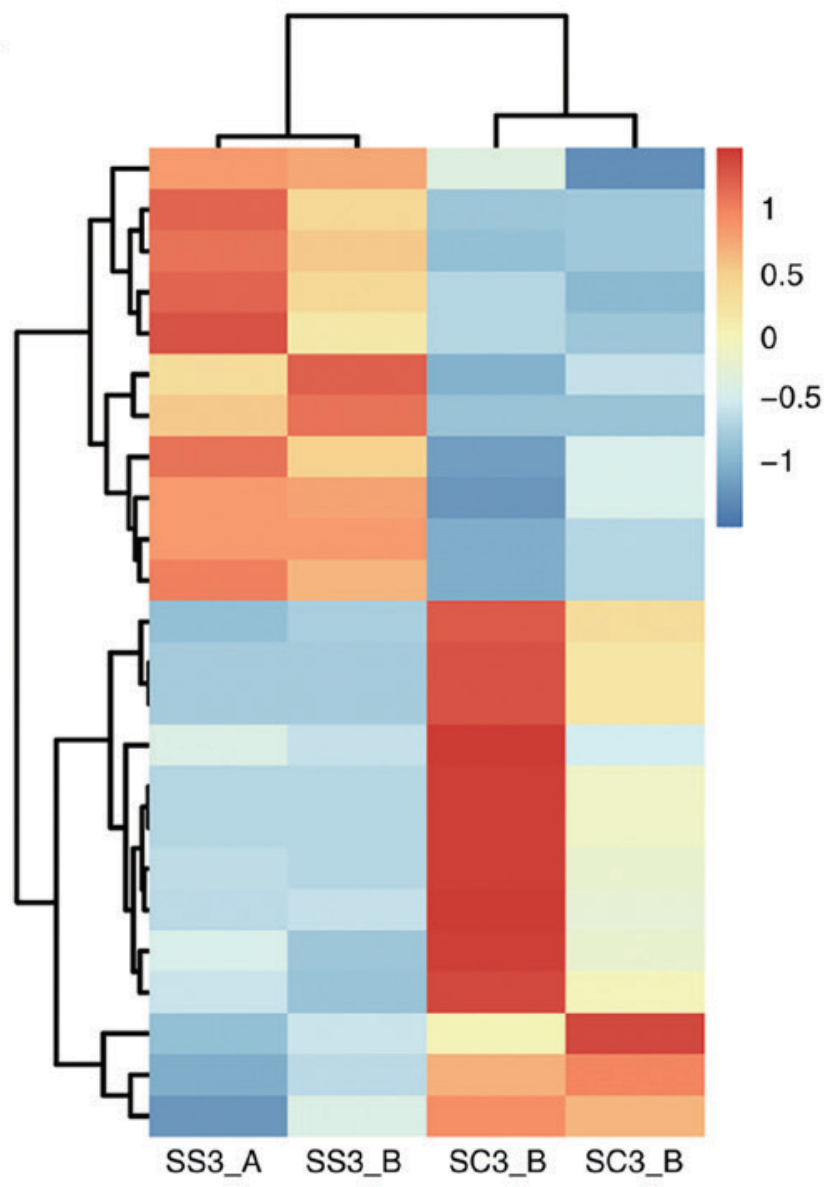

C

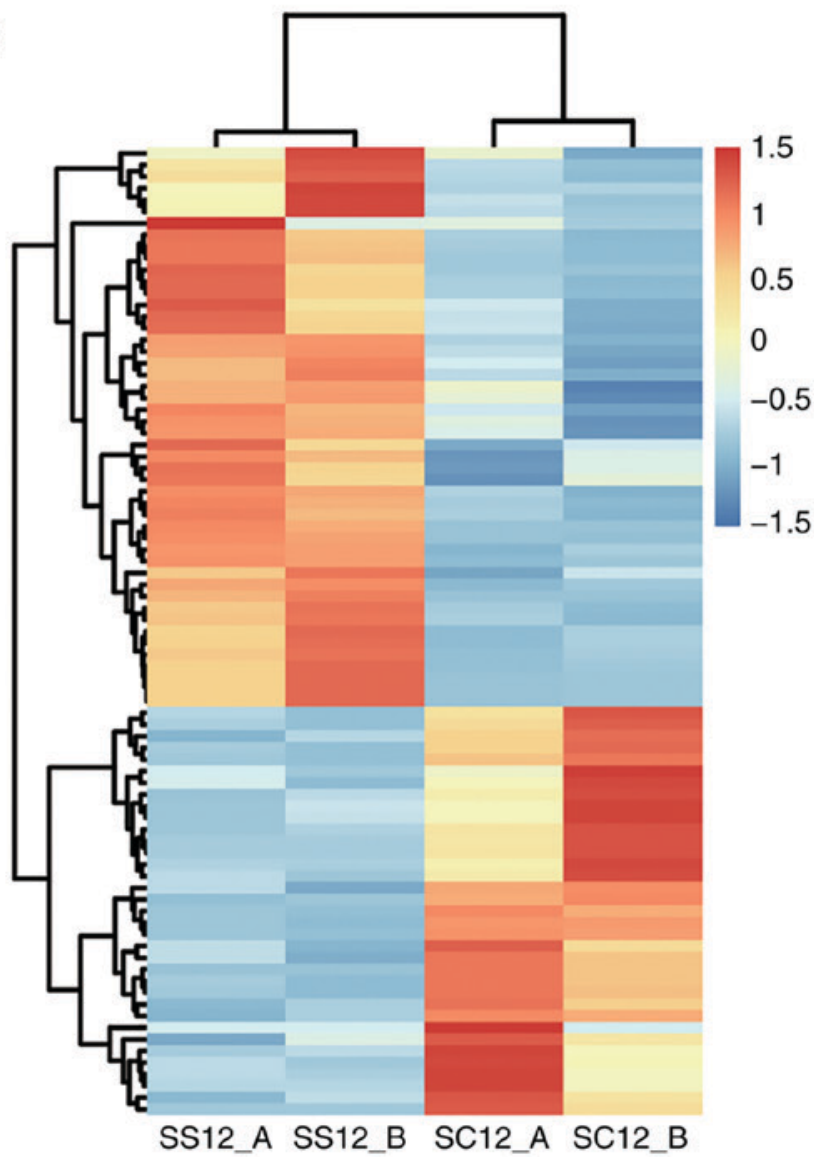

B

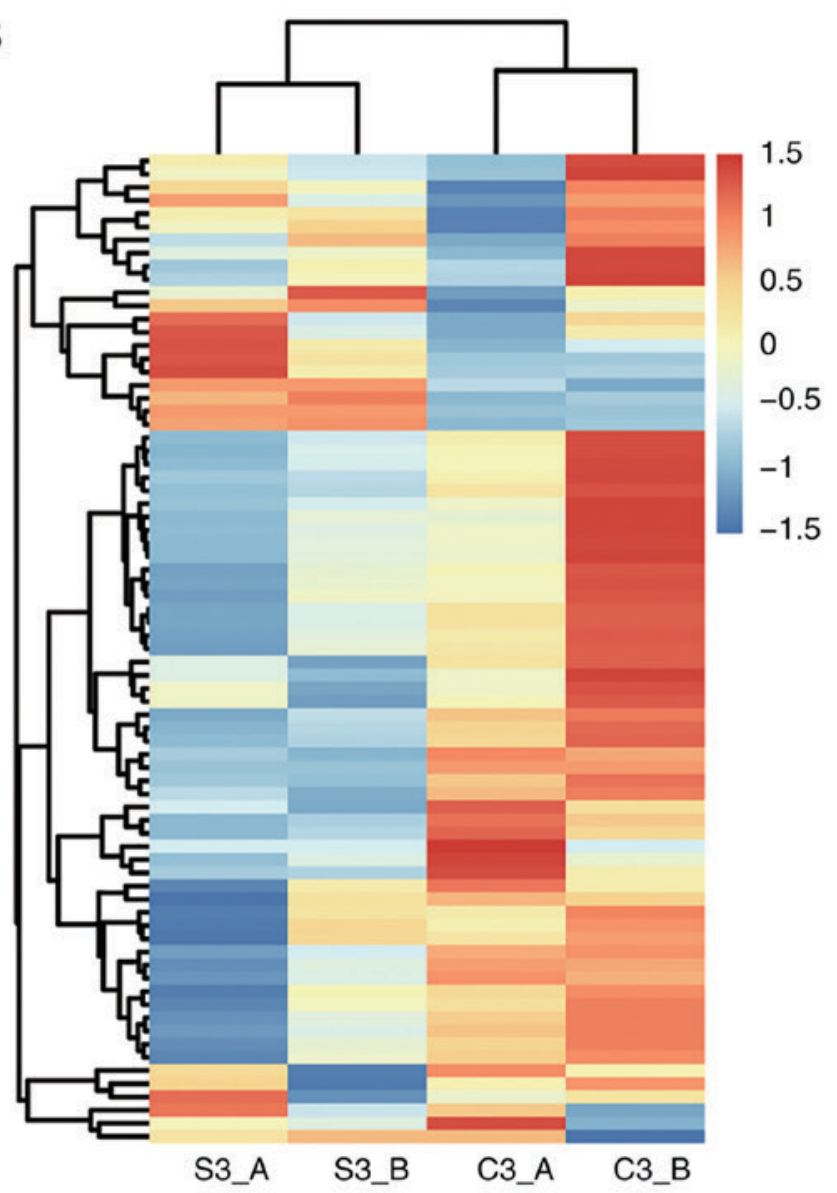

D

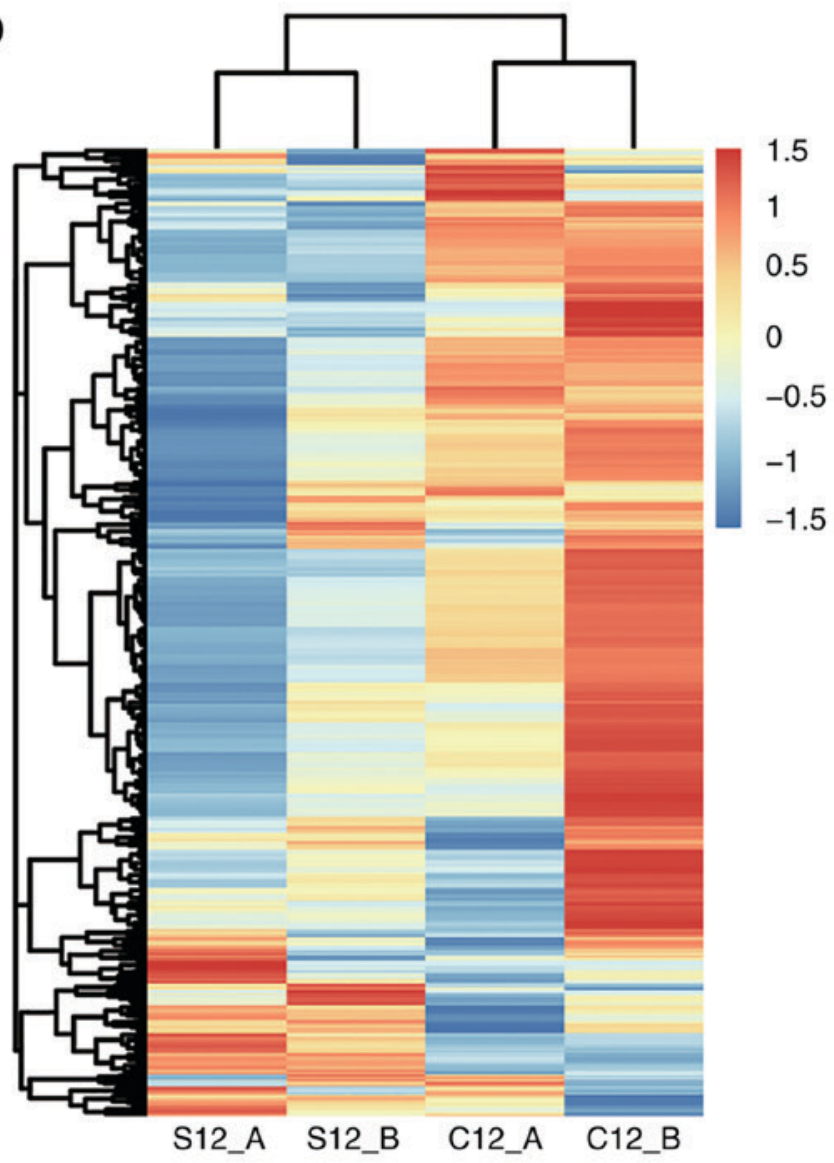

Figure 2. Prediction of DE miRNA target genes following treatment with grape seed proanthocyanidins. (A and B) Heat maps of the DE miRNAs and target genes identified in the SS3 vs. SC3. (C and D) Heat maps of the DE miRNAs and target genes identified in the SS12 vs. SC12. 
$\mathrm{E}$

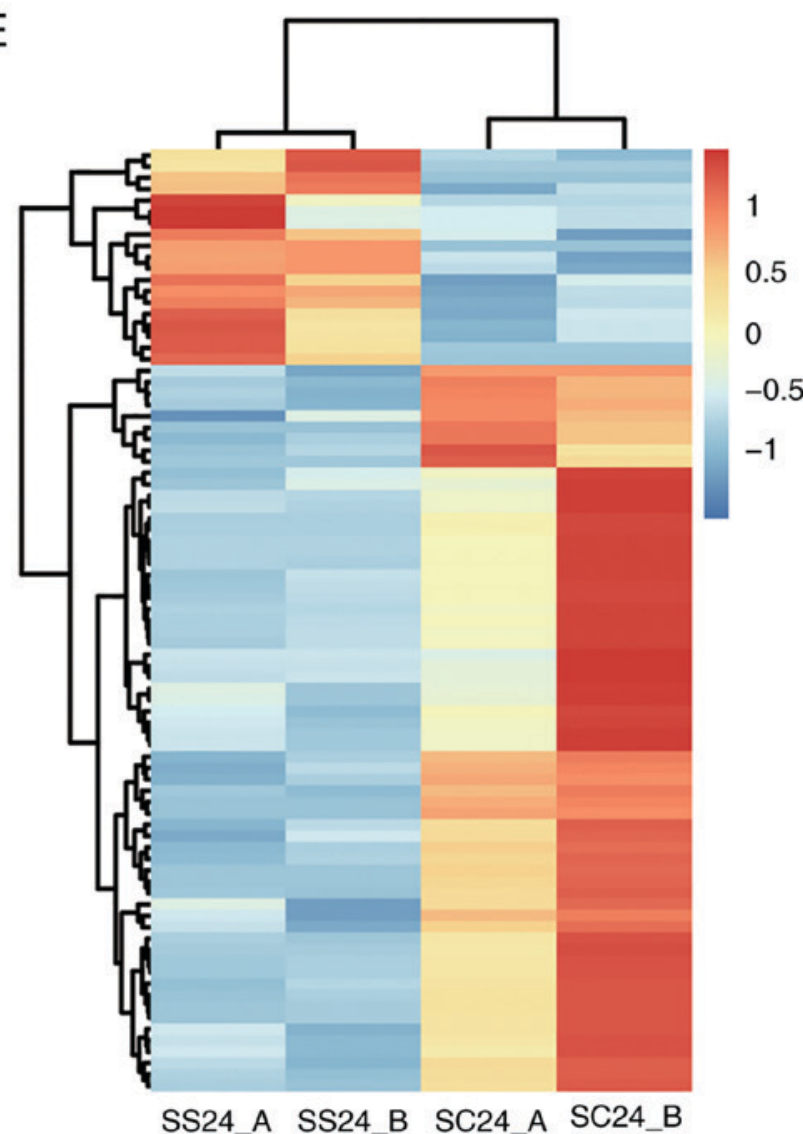

$\mathrm{F}$

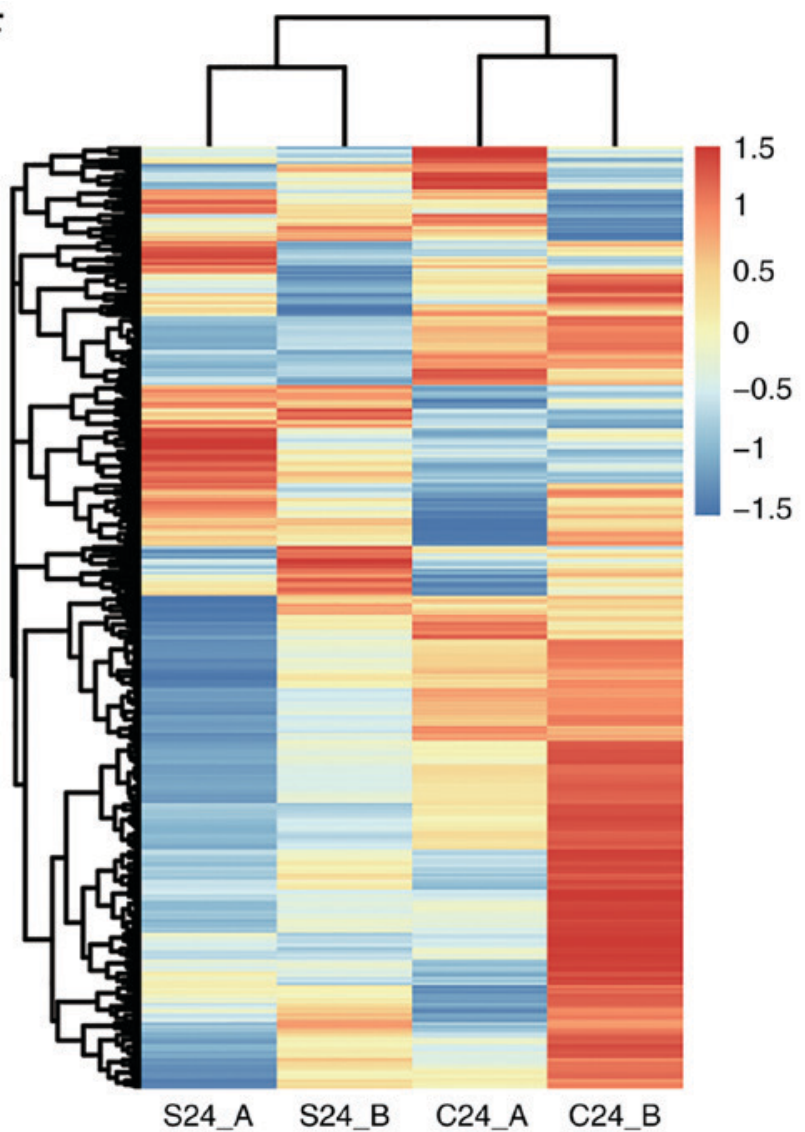

Figure 2. Continued. (E and F) Heat maps of the DE miRNAs and target genes identified in the SS24 vs. SC24. The heat maps were generated by hierarchical analysis of genes and samples, and red and blue represented upregulated and downregulated miRNAs and target genes. DE, differentially expressed; miRNA, microRNA.

A

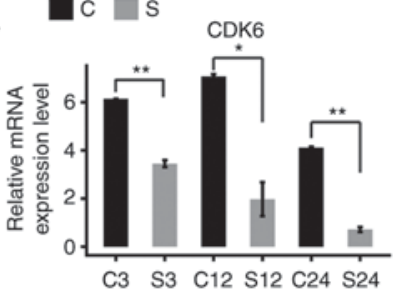

$\mathrm{B}$

$\mathrm{B} \square \mathrm{c} \square \mathrm{s}$

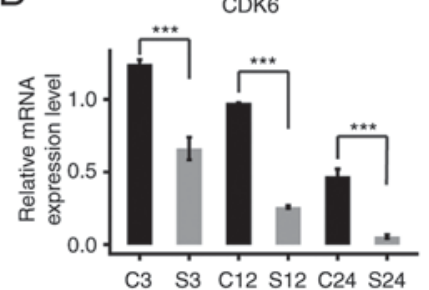

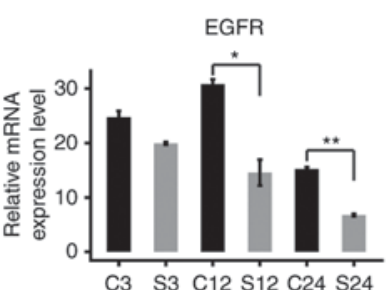

EGFR

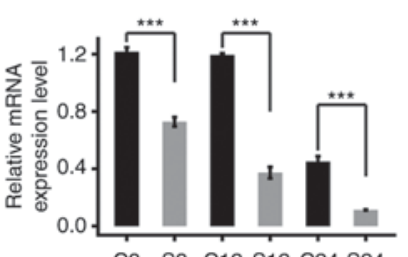

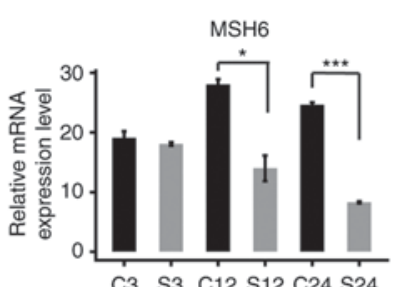

MSH6

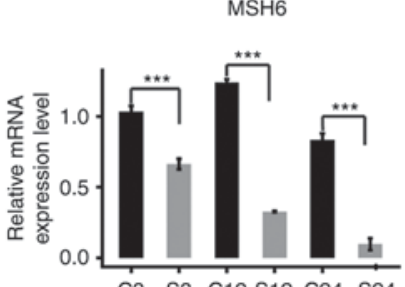

DNMT1
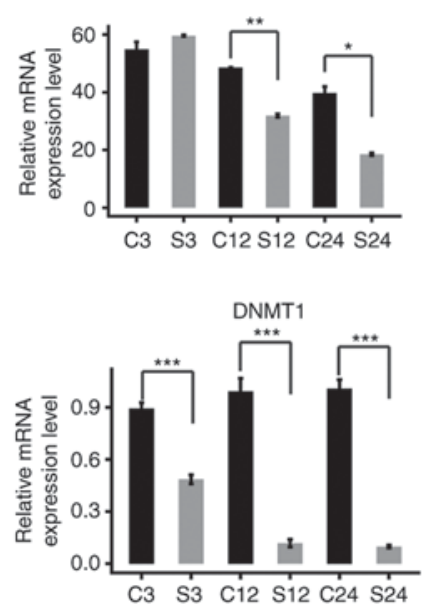

Figure 3. RT-qPCR validation of four differentially expressed microRNA target genes in GSPs-treatment and control samples. (A) mRNA expression levels of target genes were determined by high-throughput sequencing. Reads per kilobase per million mapped reads was used to calculate the expression levels of genes. (B) The mRNA expression levels of target genes were determined by RT-qPCR and normalized against GAPDH expression level. Statistical analysis was performed by Student's t-test and data are presented at the mean \pm standard deviation of three replicates. ${ }^{*} \mathrm{P}<0.05,{ }^{* *} \mathrm{P}<0.01,{ }^{* * *} \mathrm{P}<0.001$. RT-qPCR, reverse transcription-polymerase chain reaction; CDK6, cyclin dependent kinase 6; EGFR, epidermal growth factor receptor; MSH6, MutS homolog 6; DNMT1, DNA methyltransferase $1 ; \mathrm{S}$, GSPs treatment; $\mathrm{C}$, control.

identified to be correlated with 46 target genes. The negative co-expression correlations revealed between DE miRNAs and target genes demonstrated that treatment with GSPs may serve an anticancer role by regulating the expression of miRNAs.

\section{Discussion}

Phytochemicals, naturally occurring bioactive compounds, are important constituents of fruits, vegetables and legumes 
A

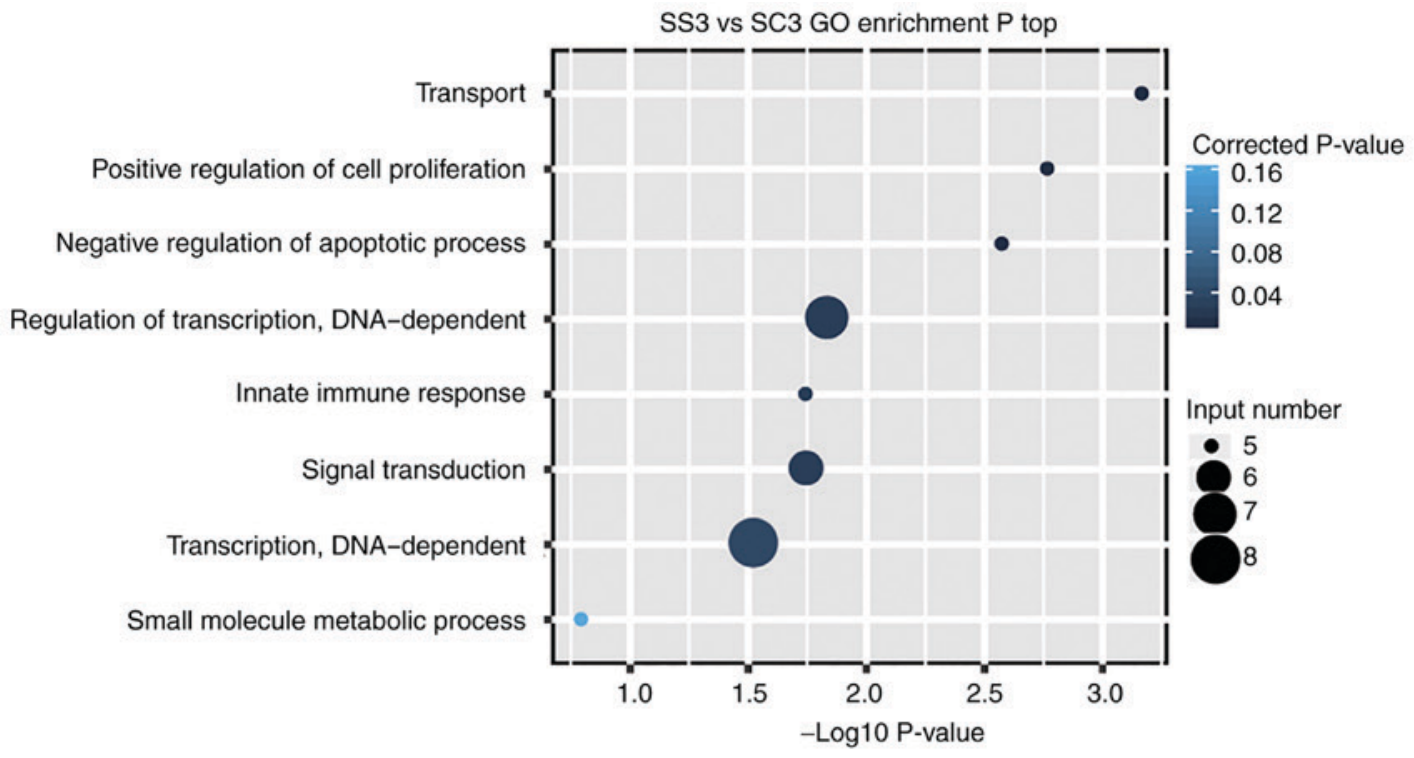

B

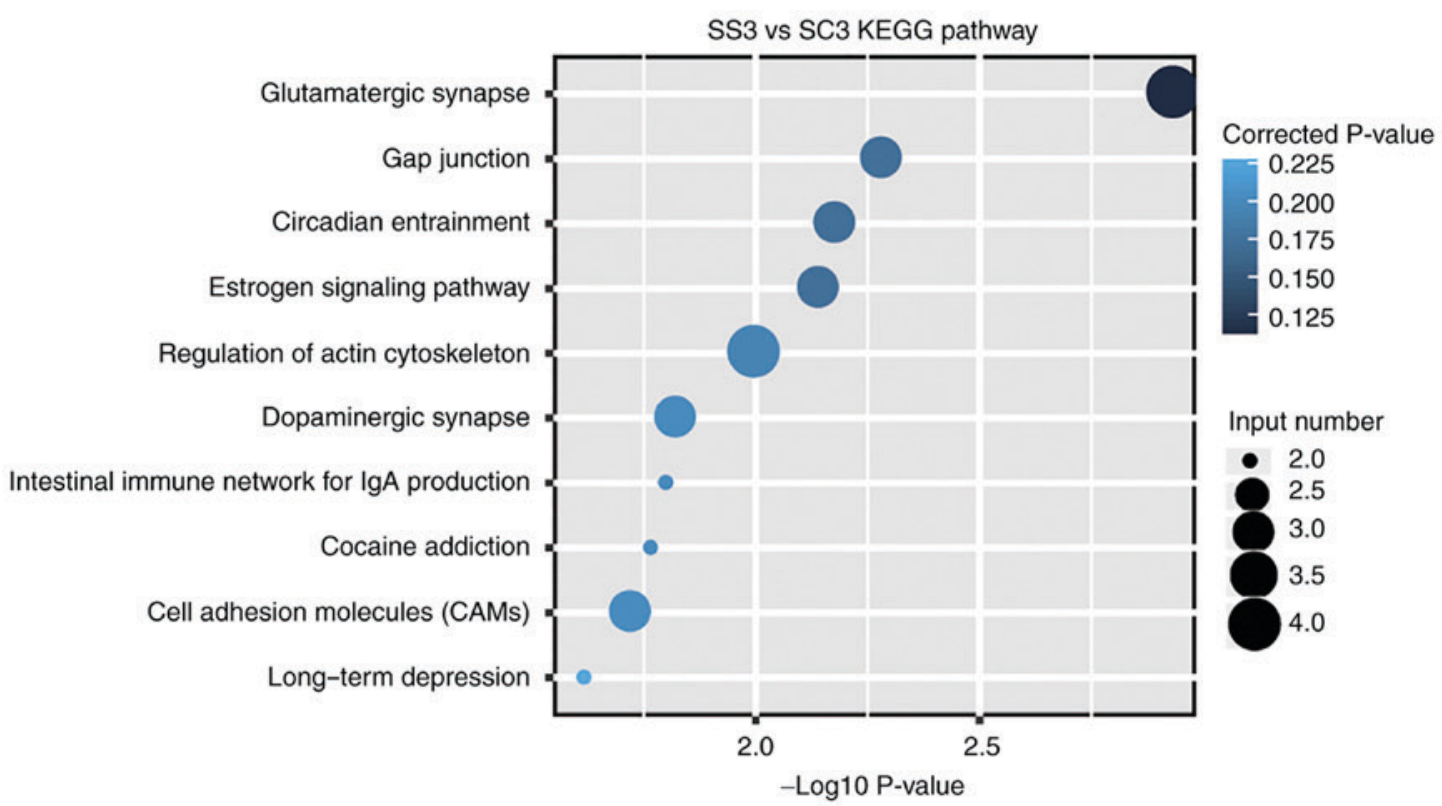

C

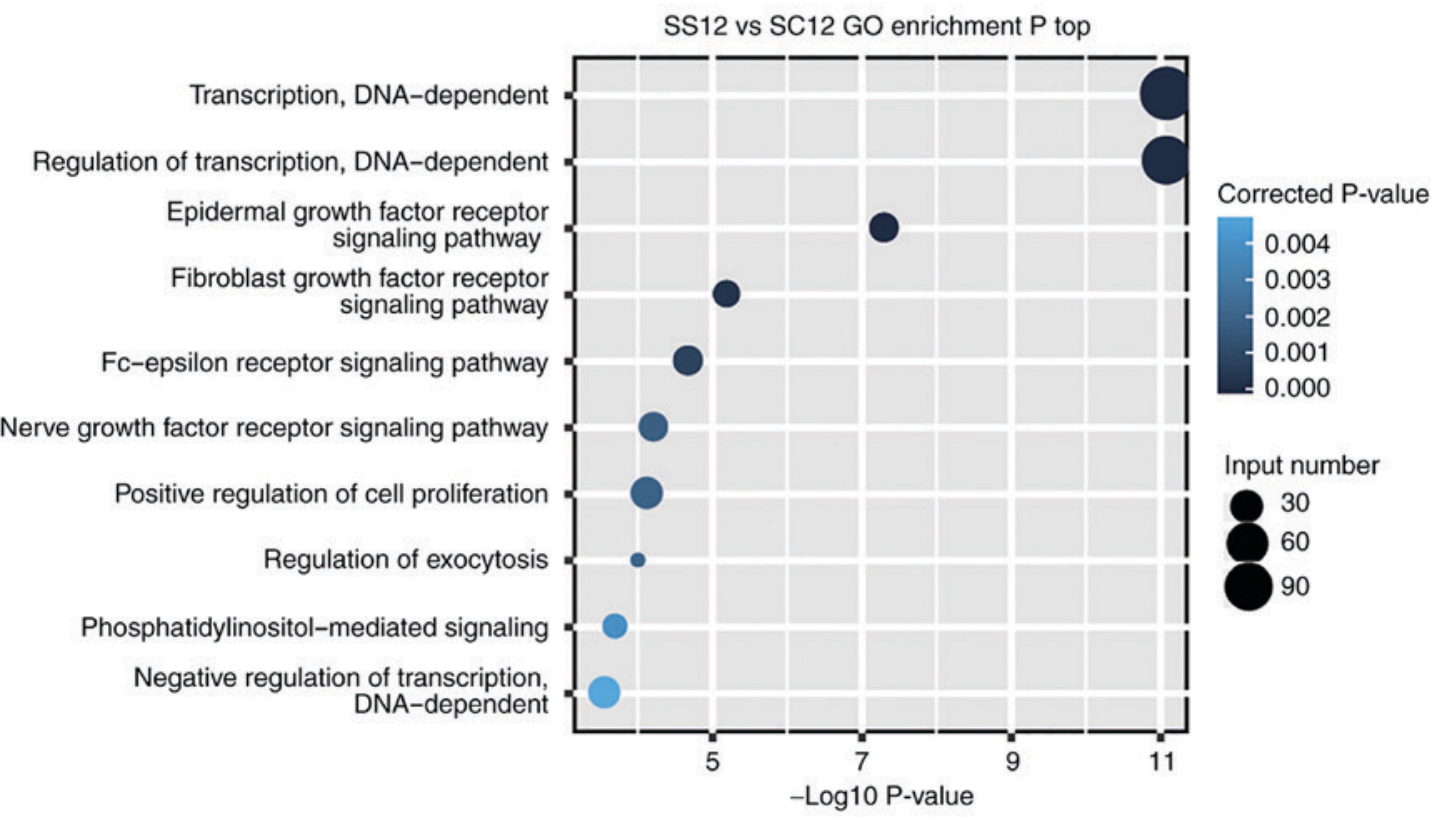

Figure 4. Functional analysis of the DE miRNA target genes. (A) GO and (B) KEGG analysis of DE miRNA target genes for SS3 vs. SC3, respectively. (C) GO analysis of DE miRNA target genes for SS12 vs. SC12. 
D

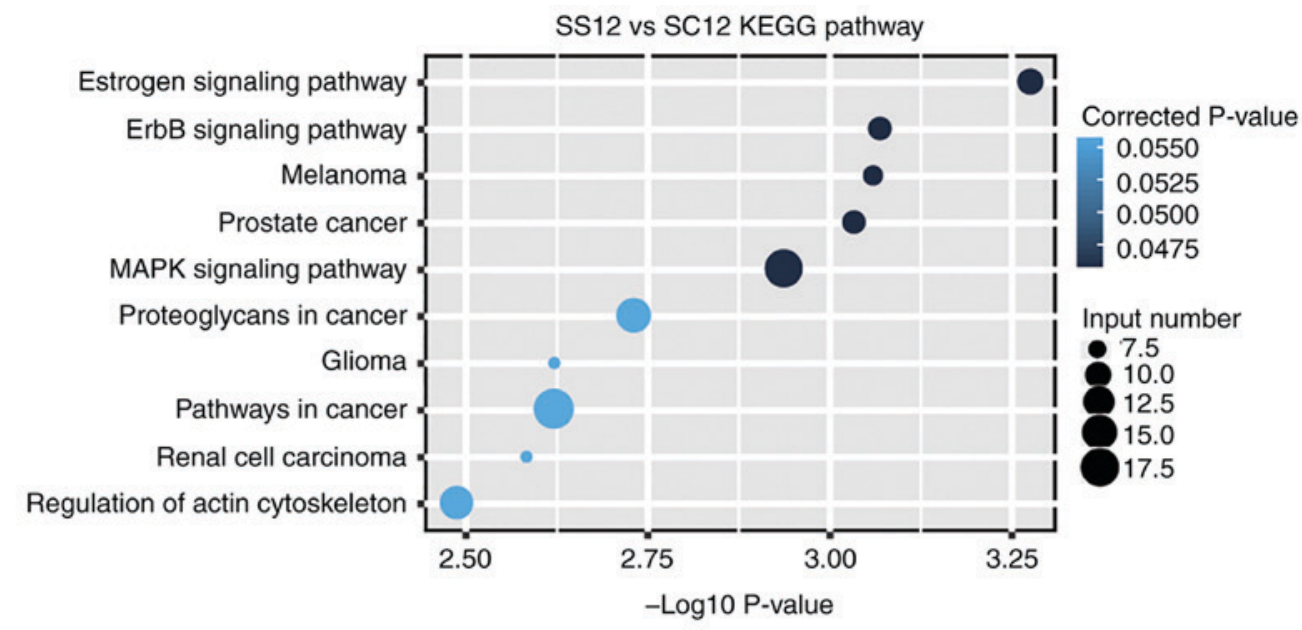

$\mathrm{E}$

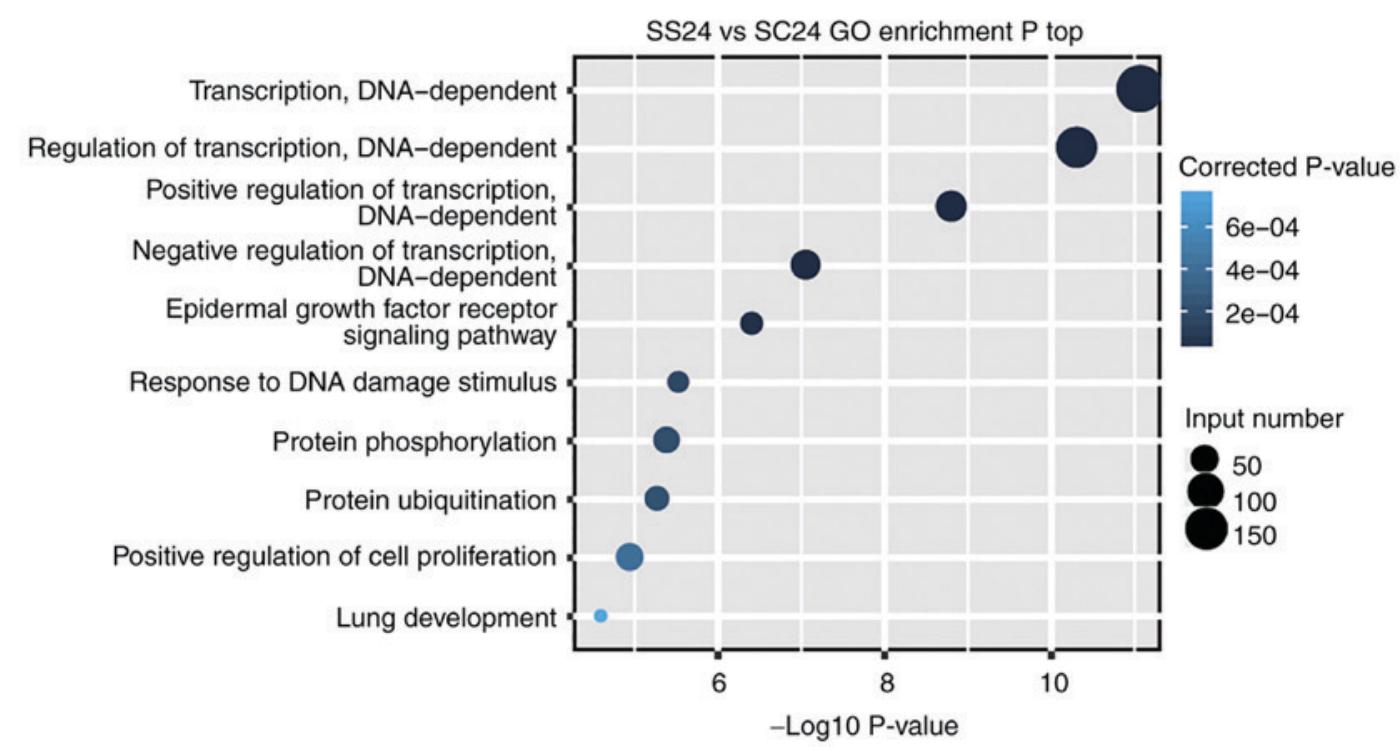

$\mathrm{F}$

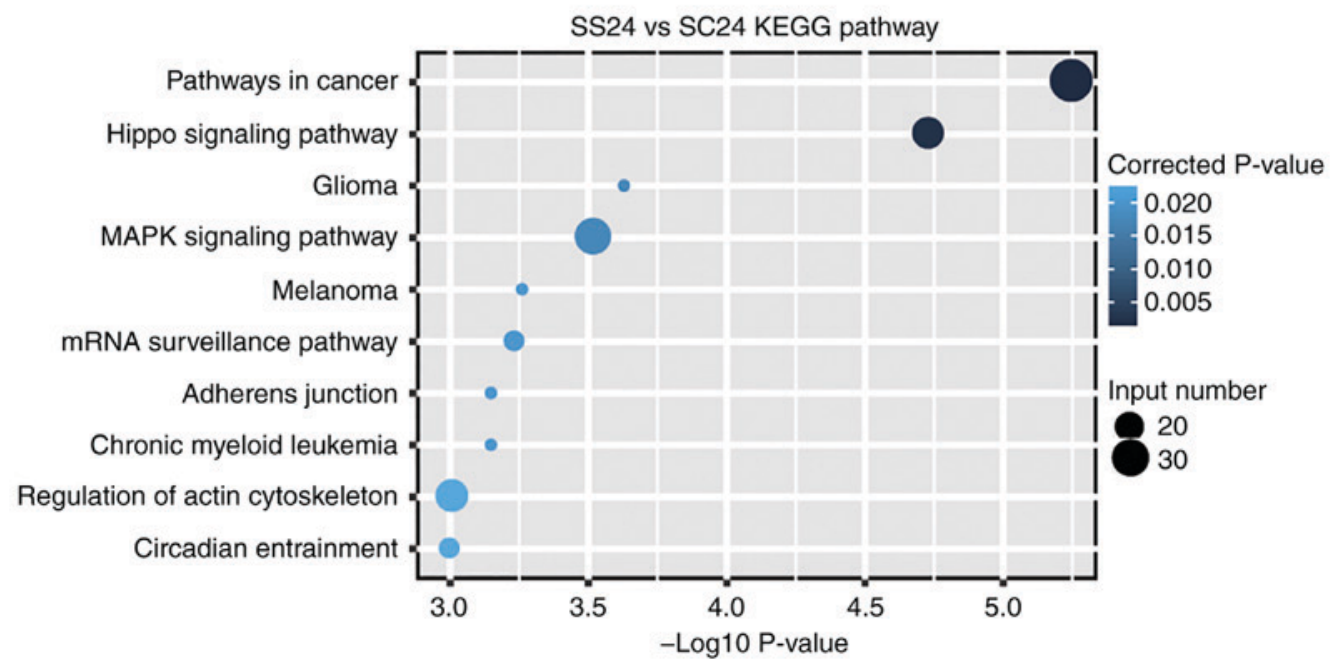

Figure 4. Continued. (D) KEGG analysis of DE miRNA target genes for SS12 vs. SC12, respectively. (E) GO and (F) KEGG analysis of DE miRNA target genes for and SS24 vs. SC24, respectively. The top ten terms are presented. DE, differentially expressed; miRNA, microRNA; GO, Gene Ontology; KEGG, Kyoto Encyclopedia of Genes and Genomes.

that provide a rich source of dietary micronutrients. Proanthocyanidins, the most common polyphenols, are the most abundant phytochemicals in the human diet and have been demonstrated to benefit human health (27). For example, proanthocyanidins have been identified to improve the symptoms of metabolic disorders, including insulin resistance, obesity, diabetes and inflammation (28). In addition, proanthocyanidins have been implicated in miRNA-based anticancer therapies (14), which are effective due to the ability of small RNAs to influence cell behavior. 


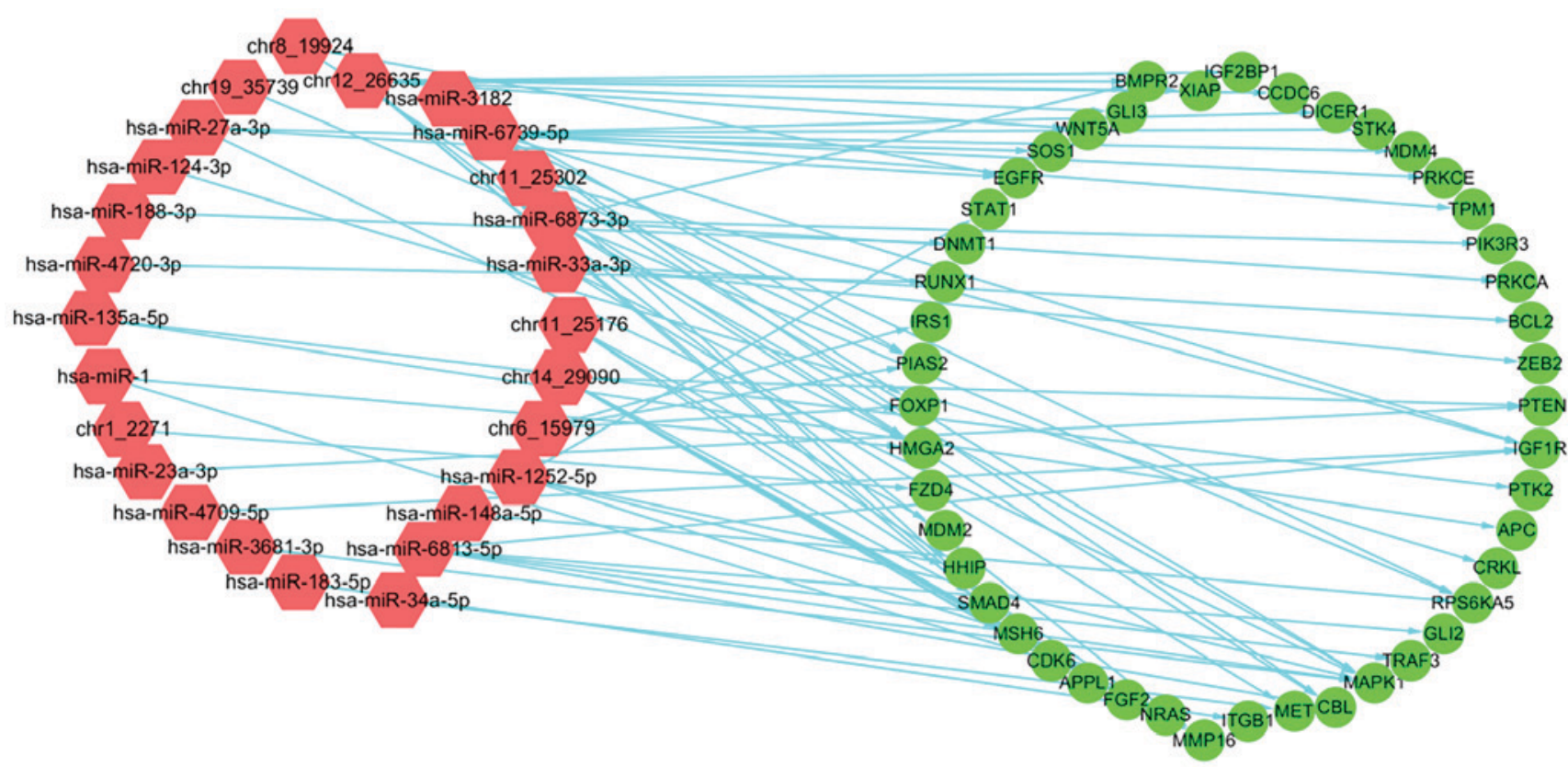

Figure 5. Interaction network analysis of DE miRNAs and target genes associated with cancer. Network analysis according to PCCs for DE miRNAs and target genes enriched in 'pathways in cancer' (ID: hsa05200), 'microRNAs in cancer' (ID: hsa05206) and 'pancreatic cancer' (ID: hsa05212). Circular nodes represent target genes and rectangular nodes signify DE miRNAs. The solid lines represent significant correlations between DE miRNAs and target genes. PPC $\geq 0.06$ and $\mathrm{P}<0.01$ indicated a statistically significant correlation. DE, differentially expressed; miRNA, microRNA; PCC, Pearson's correlation coefficient.

Proanthocyanidin extracts from a number of plants exhibit different characteristic compositions and health effects (29). GSPs, a group of proanthocyanidins that primarily contain dimers, trimers and other oligomers of catechin and epicatechin, and their galloylated esters (30), have widely been investigated and used. Previous studies have revealed that GSPs can inhibit the proliferation of cancer cells by upregulating or downregulating miRNA expression $(13,31,32)$. Furthermore, it has been demonstrated that GSPs are more effective in modulating miRNA expression compared with other proanthocyanidin extracts (13). In our previous study, it was identified that treatment with GSPs inhibited the proliferation of PC cells and certain molecular mechanisms were examined by transcriptome analysis. In the current study, 2,076 conserved miRNAs were identified and 502 novel miRNAs were revealed in PC cells. Following treatment with GSPs, the expression levels of numerous miRNAs markedly changed, which demonstrates that GSPs may serve a role in miRNA-based therapies. Additionally, multiple target genes of the DE miRNAs were associated with the proliferation of cancer cells, suggesting that treatment with GSPs may inhibit the proliferation of cancer cells by regulating the expression of miRNAs.

Notably, in SS3 vs. SC3, SS12 vs. SC12 and SS24 vs. SC24, a total of 24, 83 and 83 DE miRNAs were revealed, respectively, with only a small number of overlapping DE miRNAs. Between SS3 vs. SC3 and SS12 vs. SC12 only six overlapping DE miRNAs were identified, while only eight common DE miRNAs were revealed in SS3 vs. SC3 and SS24 vs. SC24. Furthermore between SS12 vs. SC12 and SS24 vs. SC24 only eight common DE miRNAs were identified, which was $<10 \%$ of the total number of DE miRNAs. These results indicate that the molecular mechanism underlying the regulation of miRNA expression varies at different treatment time points. This suggests that the miRNA expression response to treatment with GSPs is complicated and further investigation is required.

\section{Acknowledgements}

Not applicable.

\section{Funding}

The current study was supported by the National Natural Science Foundation of China (grant nos. 31360409, 31471667 and 31660489), Program for Young and Middle-aged Leading Talents of China Xinjiang Production and Construction Corps (grant no. 2017CB009) and the Selection and Cultivation Project of 'Talents of Xinjiang Production and Construction Corps' (Grant no. 00608017).

\section{Availability of data and materials}

The data sets analyzed during this study are available from the NCBI public repository under the accession number GSE107409 and GSE85610.

\section{Authors' contributions}

LZ, WW and ZH designed and managed the study. WW, LZ, HW and ZH drafted and revised the manuscript. YZ, YW, GM and $\mathrm{ZH}$ performed the analysis. DG, YX and MT participated in sample collection and carried out experiments. All authors read and approved the final manuscript.

\section{Ethics approval and consent to participate}

Not applicable. 


\section{Patient consent for publication}

Not applicable.

\section{Competing interests}

The authors declare that they have no competing interests.

\section{References}

1. Bartel DP: MicroRNAs: Genomics, biogenesis, mechanism, and function. Cell 116: 281-297, 2004

2. Kato M and Slack FJ: microRNAs: Small molecules with big roles-C. Elegans to human cancer. Biol Cell 100: 71-81, 2008.

3. Bartel DP: MicroRNAs: Target recognition and regulatory functions. Cell 136: 215-233, 2009.

4. Vasudevan S, Tong Y and Steitz JA: Switching from repression to activation: MicroRNAs can up-regulate translation. Science 318 1931-1934, 2007

5. Li Y, Qiu C, Tu J, Geng B, Yang J, Jiang T and Cui Q: HMDD v2.0: A database for experimentally supported human microRNA and disease associations. Nucleic Acids Res 42: D1070-D1074, 2014.

6. Kozomara A and Griffiths-Jones S: miRBase: Integrating microRNA annotation and deep-sequencing data. Nucleic Acids Res 39: D152-D157, 2011.

7. Lewis BP, Burge CB and Bartel DP: Conserved seed pairing, often flanked by adenosines, indicates that thousands of human genes are microRNA targets. Cell 120: 15-20, 2005.

8. Zhang R and Su B: Small but influential: The role of microRNAs on gene regulatory network and 3'UTR evolution. J Genet Genomics 36: 1-6, 2009.

9. Tan L, Yu JT, Tan MS, Liu QY, Wang HF, Zhang W, Jiang T and Tan L: Genome-wide serum microRNA expression profiling identifies serum biomarkers for Alzheimer's disease. J Alzheimers Dis 40: 1017-1027, 2014.

10. Dangwal S and Thum T: microRNA therapeutics in cardiovascular disease models. Annu Rev Pharmacol Toxicol 54: 185-203, 2014.

11. Iorio MV,Ferracin M, Liu CG, Veronese A, Spizzo R, Sabbioni S, Magri E, Pedriali M, Fabbri M, Campiglio M, et al: MicroRNA gene expression deregulation in human breast cancer. Cancer Res 65: 7065-7070, 2005 .

12. Cheng CJ, Bahal R, Babar IA, Pincus Z, Barrera F, Liu C, Svoronos A, Braddock DT, Glazer PM, Engelman DM, et al: MicroRNA silencing for cancer therapy targeted to the tumour microenvironment. Nature 518: 107-110, 2015.

13. Arola-Arnal A and Blade C: Proanthocyanidins modulate microRNA expression in human HepG2 cells. PLoS One 6 : e25982, 2011.

14. Bansode RR, Khatiwada JR, Losso JN and Williams LL: Targeting microRNA in cancer using plant-based proanthocyanidins. Diseases 4: E21, 2016.

15. Chung YC, Huang CC, Chen $\mathrm{CH}$, Chiang $\mathrm{HC}$, Chen $\mathrm{KB}$ Chen YJ, Liu CL, Chuang LT, Liu M and Hsu CP: Grape-seed procyanidins inhibit the in vitro growth and invasion of pancreatic carcinoma cells. Pancreas 41: 447-454, 2012.

16. Prasad R, Vaid M and Katiyar SK: Grape proanthocyanidin inhibit pancreatic cancer cell growth in vitro and in vivo through induction of apoptosis and by targeting the PI3K/Akt pathway. PLoS One 7: e43064, 2012.
17. Prasad R and Katiyar SK: Grape seed proanthocyanidins inhibit migration potential of pancreatic cancer cells by promoting mesenchymal-to-epithelial transition and targeting NF- $\kappa \mathrm{B}$. Cancer Lett 334: 118-126, 2013.

18. Wang WH, Zhan LL, Guo DQ, Xiang YJ, Zhang Y, Tian MX, and Han ZJ: Transcriptome analysis of pancreatic cancer cell response to treatment with grape seed proanthocyanidins. Oncol Lett 17: 1741-1749, 2019.

19. Langmead B, Trapnell C, Pop M and Salzberg SL: Ultrafast and memory-efficient alignment of short DNA sequences to the human genome. Genome Biol 10: R25, 2009.

20. Kozomara A and Griffiths-Jones S: miRBase: Annotating high confidence microRNAs using deep sequencing data. Nucleic Acids Res 42: D68-D73, 2014.

21. Friedlander MR, Chen W, Adamidi C, Maaskola J, Einspanier R, Knespel S and Rajewsky N: Discovering microRNAs from deep sequencing data using miRDeep. Nat Biotechnol 26: 407-415, 2008.

22. Robinson MD, McCarthy DJ and Smyth GK: edgeR: A bioconductor package for differential expression analysis of digital gene expression data. Bioinformatics 26: 139-140, 2010.

23. Krek A, Grun D, Poy MN, Wolf R, Rosenberg L, Epstein EJ, MacMenamin P, da Piedade I, Gunsalus KC, Stoffel M and Rajewsky N: Combinatorial microRNA target predictions. Nat Genet 37: 495-500, 2005.

24. Huang da W, Sherman BT and Lempicki RA: Systematic and integrative analysis of large gene lists using DAVID bioinformatics resources. Nat Protoc 4: 44-57, 2009.

25. Shannon P, Markiel A, Ozier O, Baliga NS, Wang JT, Ramage D, Amin N, Schwikowski B and Ideker T: Cytoscape: A software environment for integrated models of biomolecular interaction networks. Genome Res 13: 2498-2504, 2003.

26. Livak KJ and Schmittgen TD: Analysis of relative gene expression data using real-time quantitative PCR and the $2^{-\Delta \Delta \mathrm{CT}}$ method. Methods 25: 402-408, 2001.

27. Lee Y: Cancer chemopreventive potential of procyanidin. Toxicol Res 33: 273-282, 2017.

28. Serrano J, Puupponen-Pimia R, Dauer A, Aura AM and Saura-Calixto F: Tannins: Current knowledge of food sources, intake, bioavailability and biological effects. Mol Nutr Food Res 53 (Suppl 2): S310-S329, 2009.

29. Kidd PM: Bioavailability and activity of phytosome complexes from botanical polyphenols: The silymarin, curcumin, green tea, and grape seed extracts. Altern Med Rev 14: 226-246, 2009.

30. Bagchi D, Swaroop A, Preuss HG and Bagchi M: Free radical scavenging, antioxidant and cancer chemoprevention by grape seed proanthocyanidin: An overview. Mutat Res 768: 69-73, 2014.

31. Ma J, Fang B, Ma C, Pang H, Zeng F and Xia J: Proanthocyanidins inhibit pancreatic cancer AsPC-1 cell growth and migration through up-regulation of let-7a. Nan Fang Yi Ke Da Xue Xue Bao 35: 1110-1115, 2015 (In Chinese).

32. Prasad R and Katiyar SK: Down-regulation of miRNA-106b inhibits growth of melanoma cells by promoting G1-phase cell cycle arrest and reactivation of p21/WAF1/Cip1 protein. Oncotarget 5: 10636-10649, 2014.

This work is licensed under a Creative Commons Attribution-NonCommercial-NoDerivatives 4.0 International (CC BY-NC-ND 4.0) License. 